Pacific

Journal of

Mathematics

NEW WEIGHTED INTEGRAL INEQUALITIES FOR DIFFERENTIAL FORMS IN SOME DOMAINS

SHUSEN Ding 


\title{
NEW WEIGHTED INTEGRAL INEQUALITIES FOR DIFFERENTIAL FORMS IN SOME DOMAINS
}

\author{
Shusen Ding
}

\begin{abstract}
We first prove local weighted integral inequalities for differential forms. Then, as applications of our local results, we prove global weighted integral inequalities for differential forms in $L^{s}(\mu)$-averaging domains and John domains, respectively, which can be considered as generalizations of the classical Poincaré-type inequality.
\end{abstract}

\section{Introduction.}

Differential forms are interesting and important generalizations of real functions and distributions. Many interesting results and applications of differential forms have recently been found in some fields, such as tensor analysis, potential theory, partial differential equations and quasiregular mappings, see $[\mathbf{B}],[\mathbf{C}],[\mathbf{D} \mathbf{1}],[\mathbf{H K M}],[\mathbf{I}],[\mathbf{I L}]$ and $[\mathbf{I M}]$. In many cases, we need to know the integrability of differential forms and estimate the integrals for differential forms. In this paper we prove local weighted Poincaré-type inequalities for differential forms in any kind of domains and global weighted Poincaré-type inequalities for differential forms in John domains and $L^{s}(\mu)$ averaging domains, where $\mu$ is a measure defined by $d \mu=w(x) d x$ and $w \in A_{r}^{\lambda}$. These integral inequalities can be used to study the integrability of differential forms and estimate the integrals for differential forms. As we know, $A$-harmonic tensors are the special differential forms which are solutions to the $A$-harmonic equation for differential forms: $d^{\star} A(x, d u)=0$, where $A: \Omega \times \wedge^{l}\left(\mathbf{R}^{n}\right) \rightarrow \wedge^{l}\left(\mathbf{R}^{n}\right)$ is an operator satisfying some conditions, see $[\mathbf{I}],[\mathbf{I L}]$ and $[\mathbf{N}]$. So that all of the results about differential forms in this paper remain true for $A$-harmonic tensors. Therefore, our new results concerning differential forms are of interest in some fields, such as those mentioned above.

Throughout this paper, we always assume $\Omega$ is a connected open subset of $\mathbf{R}^{n}$. Let $e_{1}, e_{2}, \ldots, e_{n}$ denote the standard unit basis of $\mathbf{R}^{n}$. For $l=$ $0,1, \ldots, n$, the linear space of $l$-vectors, spanned by the exterior products $e_{I}=e_{i_{1}} \wedge e_{i_{2}} \wedge \cdots e_{i_{l}}$, corresponding to all ordered $l$-tuples $I=\left(i_{1}, i_{2}, \ldots, i_{l}\right)$, $1 \leq i_{1}<i_{2}<\cdots<i_{l} \leq n$, is denoted by $\wedge^{l}=\wedge^{l}\left(\mathbf{R}^{n}\right)$. The Grassman algebra $\wedge=\oplus \wedge^{l}$ is a graded algebra with respect to the exterior products. For $\alpha=\sum \alpha^{I} e_{I} \in \wedge$ and $\beta=\sum \beta^{I} e_{I} \in \wedge$, the inner product in $\wedge$ is given 
by $\langle\alpha, \beta\rangle=\sum \alpha^{I} \beta^{I}$ with summation over all $l$-tuples $I=\left(i_{1}, i_{2}, \ldots, i_{l}\right)$ and all integers $l=0,1, \ldots, n$. We define the Hodge star operator $\star$ : $\wedge \rightarrow \wedge$ by the rule $\star 1=e_{1} \wedge e_{2} \wedge \cdots \wedge e_{n}$ and $\alpha \wedge \star \beta=\beta \wedge \star \alpha=\langle\alpha, \beta\rangle(\star 1)$ for all $\alpha, \beta \in \wedge$. Hence the norm of $\alpha \in \wedge$ is given by the formula $|\alpha|^{2}=\langle\alpha, \alpha\rangle=$ $\star(\alpha \wedge \star \alpha) \in \wedge^{0}=\mathbf{R}$. The Hodge star is an isometric isomorphism on $\wedge$ with $\star: \wedge^{l} \rightarrow \wedge^{n-l}$ and $\star \star(-1)^{l(n-l)}: \wedge^{l} \rightarrow \wedge^{l}$. Let $0<p<\infty$, we denote the weighted $L^{p}$-norm of a measurable function $f$ over $E$ by

$$
\|f\|_{p, E, w}=\left(\int_{E}|f(x)|^{p} w(x) d x\right)^{1 / p} .
$$

As we know, a differential $l$-form $\omega$ on $\Omega$ is a Schwartz distribution on $\Omega$ with values in $\wedge^{l}\left(\mathbf{R}^{n}\right)$. In particular, for $l=0, \omega$ is a real function or a distribution. We denote the space of differential $l$-forms by $D^{\prime}\left(\Omega, \wedge^{l}\right)$. We write $L^{p}\left(\Omega, \wedge^{l}\right)$ for the $l$-forms $\omega(x)=\sum_{I} \omega_{I}(x) d x_{I}=\sum \omega_{i_{1} i_{2} \cdots i_{l}}(x) d x_{i_{1}} \wedge$ $d x_{i_{2}} \wedge \cdots \wedge d x_{i_{l}}$ with $\omega_{I} \in L^{p}(\Omega, \mathbf{R})$ for all ordered $l$-tuples $I$. Thus $L^{p}\left(\Omega, \wedge^{l}\right)$ is a Banach space with norm

$$
\|\omega\|_{p, \Omega}=\left(\int_{\Omega}|\omega(x)|^{p} d x\right)^{1 / p}=\left(\int_{\Omega}\left(\sum_{I}\left|\omega_{I}(x)\right|^{2}\right)^{p / 2} d x\right)^{1 / p}
$$

Similarly, $W_{p}^{1}\left(\Omega, \wedge^{l}\right)$ are those differential $l$-forms on $\Omega$ whose coefficients are in $W_{p}^{1}(\Omega, \mathbf{R})$. The notations $W_{p, \text { loc }}^{1}(\Omega, \mathbf{R})$ and $W_{p, \text { loc }}^{1}\left(\Omega, \wedge^{l}\right)$ are selfexplanatory. We denote the exterior derivative by $d: D^{\prime}\left(\Omega, \wedge^{l}\right) \rightarrow D^{\prime}\left(\Omega, \wedge^{l+1}\right)$ for $l=0,1, \ldots, n$. Its formal adjoint operator $d^{\star}: D^{\prime}\left(\Omega, \wedge^{l+1}\right) \rightarrow D^{\prime}\left(\Omega, \wedge^{l}\right)$ is given by $d^{\star}=(-1)^{n l+1} \star d \star$ on $D^{\prime}\left(\Omega, \wedge^{l+1}\right), l=0,1, \ldots, n$.

We write $\mathbf{R}=\mathbf{R}^{1}$. Balls are denoted by $B$ and $\sigma B$ is the ball with the same center as $B$ and with $\operatorname{diam}(\sigma B)=\sigma \operatorname{diam}(B)$. The $n$-dimensional Lebesgue measure of a set $E \subseteq \mathbf{R}^{n}$ is denoted by $|E|$. We call $w$ a weight if $w \in L_{\text {loc }}^{1}\left(\mathbf{R}^{n}\right)$ and $w>0$ a.e. Also in general $d \mu=w d x$ where $w$ is a weight. The following result appears in $[\mathbf{I L}]$ : Let $Q \subset \mathbf{R}^{n}$ be a cube or a ball. To each $y \in Q$ there corresponds a linear operator $K_{y}: C^{\infty}\left(Q, \wedge^{l}\right) \rightarrow C^{\infty}\left(Q, \wedge^{l-1}\right)$ defined by

$$
\left(K_{y} \omega\right)\left(x ; \xi_{1}, \ldots, \xi_{l}\right)=\int_{0}^{1} t^{l-1} \omega\left(t x+y-t y ; x-y, \xi_{1}, \ldots, \xi_{l-1}\right) d t
$$

and the decomposition

$$
\omega=d\left(K_{y} \omega\right)+K_{y}(d \omega)
$$

We define another linear operator $T_{Q}: C^{\infty}\left(Q, \wedge^{l}\right) \rightarrow C^{\infty}\left(Q, \wedge^{l-1}\right)$ by averaging $K_{y}$ over all points $y$ in $Q$

$$
T_{Q} \omega=\int_{Q} \varphi(y) K_{y} \omega d y
$$


where $\varphi \in C_{0}^{\infty}(Q)$ is normalized by $\int_{Q} \varphi(y) d y=1$. We define the $l$-form $\omega_{Q} \in D^{\prime}\left(Q, \wedge^{l}\right)$ by

$$
\omega_{Q}=|Q|^{-1} \int_{Q} \omega(y) d y, \quad l=0, \quad \text { and } \omega_{Q}=d\left(T_{Q} \omega\right), \quad l=1,2, \ldots, n,
$$

for all $\omega \in L^{p}\left(Q, \wedge^{l}\right), 1 \leq p<\infty$.

\section{Local weighted integral inequalities.}

Definition 2.1. We say the weight $w(x)>0$ satisfies the $A_{r}^{\lambda}$-condition, $r>1$ and $\lambda>0$, and write $w \in A_{r}^{\lambda}$, if

$$
\sup _{B}\left(\frac{1}{|B|} \int_{B} w d x\right)\left(\frac{1}{|B|} \int_{B} w^{1 /(1-r)} d x\right)^{\lambda(r-1)}<\infty
$$

for any ball $B \subset \mathbf{R}^{n}$.

The following generalized Hölder's inequality will be used repeatedly.

Lemma 2.2. Let $0<\alpha<\infty, 0<\beta<\infty$ and $s^{-1}=\alpha^{-1}+\beta^{-1}$. If $f$ and $g$ are measurable functions on $\mathbf{R}^{n}$, then

$$
\|f g\|_{s, \Omega} \leq\|f\|_{\alpha, \Omega} \cdot\|g\|_{\beta, \Omega}
$$

for any $\Omega \subset \mathbf{R}^{n}$.

We also need the following lemma $[\mathbf{G}]$.

Lemma 2.3. If $w \in A_{r}^{1}, r>1$, then there exist constants $\beta>1$ and $C$, independent of $w$, such that

$$
\|w\|_{\beta, Q} \leq C|Q|^{(1-\beta) / \beta}\|w\|_{1, Q}
$$

for any cube or any ball $Q \subset \mathbf{R}^{n}$.

The following version of the Poincaré inequality appears in $[\mathbf{N}]$.

Lemma 2.4. Let $u \in D^{\prime}\left(Q, \wedge^{l}\right)$ and $d u \in L^{p}\left(Q, \wedge^{l+1}\right)$. Then $u-u_{Q}$ is in $W_{p}^{1}\left(Q, \wedge^{l}\right)$ with $1<p<\infty$ and

$$
\left\|u-u_{Q}\right\|_{p, Q} \leq C(n, p)|Q|^{1 / n}\|d u\|_{p, Q}
$$

for $Q$ a cube or a ball in $\mathbf{R}^{n}, l=0,1, \ldots, n$.

Different versions of the classical Poincaré inequality have been established in the study of the Sobolev space and differential forms, see $[\mathbf{C}],[\mathbf{S}]$ and $\left[\mathbf{I L}\right.$. Susan G. Staples proves the Poincaré inequality in $L^{s}$-averaging domains in $[\mathbf{S}]$. Tadeusz Iwaniec and Adam Lutoborski prove the following local Poincaré-type inequality in $[\mathbf{I L}]$ which plays a crucial rule in generalizing the theory of Sobolev functions to differential forms. 
Lemma 2.5. Let $u \in D^{\prime}\left(Q, \wedge^{l}\right)$ and $d u \in L^{p}\left(Q, \wedge^{l+1}\right)$. Then $u-u_{Q}$ is in $L^{n p /(n-p)}\left(Q, \wedge^{l}\right)$ and

$$
\left(\int_{Q}\left|u-u_{Q}\right|^{n p /(n-p)} d x\right)^{(n-p) / n p} \leq C_{p}(n)\left(\int_{Q}|d u|^{p} d x\right)^{1 / p}
$$

for $Q$ a cube or a ball in $\mathbf{R}^{n}, l=0,1, \ldots, n$ and $1<p<n$.

We now prove the following version of the local weighted Poincaré-type inequality for differential forms.

Theorem 2.6. Let $u \in D^{\prime}\left(B, \wedge^{l}\right)$ and $d u \in L^{p}\left(B, \wedge^{l+1}\right), l=0,1, \ldots, n$. Assume that $1<s<p<\infty$. Then exists a constant $\beta>1$ such that if $w \in A_{r}^{1} \cap A_{p / k}^{s / p}$ for some $r>1$ and $k$ with $s \beta /(\beta-1) \leq k<p$, we have

$$
\left(\frac{1}{|B|} \int_{B}\left|u-u_{B}\right|^{s} w d x\right)^{1 / s} \leq C|B|^{1 / n}\left(\frac{1}{|B|} \int_{B}|d u|^{p} w d x\right)^{1 / p}
$$

for all balls $B \subset \mathbf{R}^{n}$. Here $C$ is a constant independent of $u$ and $d u$.

Proof. Since $w \in A_{r}^{1}$ for some $r>1$, by Lemma 2.3, there exist constants $\beta>1$ and $C_{1}>0$, such that

$$
\|w\|_{\beta, B} \leq C_{1}|B|^{(1-\beta) / \beta}\|w\|_{1, B}
$$

for any cube or any ball $B \subset \mathbf{R}^{n}$. Choose $t=s \beta /(\beta-1)$, then $1<s<t$ and $\beta=t /(t-s)$. Since $1 / s=1 / t+(t-s) / s t$, by Hölder's inequality, Lemma 2.4 and (2.8), we have

$$
\begin{aligned}
\left\|u-u_{B}\right\|_{s, B, w} & =\left(\int_{B}\left(\left|u-u_{B}\right| w^{1 / s}\right)^{s} d x\right)^{1 / s} \\
& \leq\left(\int_{B}\left|u-u_{B}\right|^{t} d x\right)^{1 / t}\left(\int_{B}\left(w^{1 / s}\right)^{s t /(t-s)} d x\right)^{(t-s) / s t} \\
& =\left\|u-u_{B}\right\|_{t, B} \cdot\left(\int_{B} w^{t /(t-s)} d x\right)^{(t-s) / s t} \\
& \leq C_{2}|B|^{(1-\beta) / \beta s}\|w\|_{1, B}^{1 / s} \cdot\left\|u-u_{B}\right\|_{t, B} \\
& \leq C_{2}|B|^{(1-\beta) / \beta s}\|w\|_{1, B}^{1 / s} \cdot C_{3}|B|^{1 / n}\|d u\|_{t, B} \\
& =C_{4}|B|^{1 / n}|B|^{(1-\beta) / \beta s}\|w\|_{1, B}^{1 / s} \cdot\|d u\|_{t, B}
\end{aligned}
$$

Now $t=s \beta /(\beta-1)<p$ and $1 / t=1 / p+(p-t) / p t$, by Hölder's inequality again we obtain

$$
\|d u\|_{t, B}=\left(\int_{B}|d u|^{t} d x\right)^{1 / t}
$$




$$
\begin{aligned}
& =\left(\int_{B}\left(|d u| w^{1 / p} w^{-1 / p}\right)^{t} d x\right)^{1 / t} \\
& \leq\left(\int_{B}\left(|d u| w^{1 / p}\right)^{p} d x\right)^{1 / p}\left(\int_{B}\left(\frac{1}{w}\right)^{t /(p-t)} d x\right)^{(p-t) / p t} \\
& =\|1 / w\|_{t /(p-t), B}^{1 / p}\left(\int_{B}|d u|^{p} w d x\right)^{1 / p} .
\end{aligned}
$$

Combining (2.9) and (2.10) yields

(2.11) $\quad\left\|u-u_{B}\right\|_{s, B, w}$

$$
\leq C_{4}|B|^{1 / n+(1-\beta) / \beta s}\|w\|_{1, B}^{1 / s} \cdot\|1 / w\|_{t /(p-t), B}^{1 / p} \cdot\left(\int_{B}|d u|^{p} w d x\right)^{1 / p} .
$$

Note $t=s \beta /(\beta-1) \leq k$, then $p / k \leq p / t$. By Theorem 2.4 in [D2], we find that $w \in A_{p / k}^{s / p} \subset A_{p / t}^{s / p}$. Therefore, we have

$$
\begin{aligned}
& \|w\|_{1, B}^{1 / s} \cdot\|1 / w\|_{t /(p-t), B}^{1 / p} \\
& =\left(\int_{B} w d x\right)^{1 / s}\left(\int_{B}\left(\frac{1}{w}\right)^{t /(p-t)} d x\right)^{(p-t) / p t} \\
& =\left(\left(\int_{B} w d x\right)\left(\int_{B}\left(\frac{1}{w}\right)^{t /(p-t)} d x\right)^{s(p-t) / p t}\right)^{1 / s} \\
& =\left(|B|^{1+s(p-t) / p t}\right)^{1 / s} \\
& \left(\left(\frac{1}{|B|} \int_{B} w d x\right)\left(\frac{1}{|B|} \int_{B}\left(\frac{1}{w}\right)^{1 /(p / t-1)} d x\right)^{(s / p)(p / t-1)}\right)^{1 / s} \\
& \leq C_{5}|B|^{1 / s+1 / t-1 / p} \text {. }
\end{aligned}
$$

Substituting (2.12) in (2.11) implies

$$
\left\|u-u_{B}\right\|_{s, B, w} \leq C_{6}|B|^{1 / n+1 / s-1 / p}\left(\int_{B}|d u|^{p} w d x\right)^{1 / p} .
$$

We can write $(2.13)$ as

$$
\left(\frac{1}{|B|} \int_{B}\left|u-u_{B}\right|^{s} w(x) d x\right)^{1 / s} \leq C|B|^{1 / n}\left(\frac{1}{|B|} \int_{B}|d u|^{p} w d x\right)^{1 / p} .
$$

This ends the proof of Theorem 2.6.

We now prove other versions of the local weighted Poincaré-type inequality for differential forms. 
Theorem 2.14. Let $u \in D^{\prime}\left(B, \wedge^{l}\right)$ and $d u \in L^{n}\left(B, \wedge^{l+1}\right), l=0,1, \ldots, n$. Then there exists a constant $\beta>1$ such that if $w \in A_{r}^{1} \cap A_{n / s}^{s / n}$, where $s=n / \beta$ and $r>1$, we have

$$
\left(\frac{1}{|B|} \int_{B}\left|u-u_{B}\right|^{s} w d x\right)^{1 / s} \leq C|B|^{1 / n}\left(\frac{1}{|B|} \int_{B}|d u|^{n} w d x\right)^{1 / n}
$$

for all balls $B \subset \mathbf{R}^{n}$. Here $C$ is a constant independent of $u$ and $d u$.

Note that we can write $(2.15)$ as

$$
\left(\frac{1}{|B|} \int_{B}\left|u-u_{B}\right|^{s} d \mu\right)^{1 / s} \leq C\left(\int_{B}|d u|^{n} d \mu\right)^{1 / n},
$$

where $d \mu=w(x) d x$.

Proof. Since $w \in A_{r}^{1}, r>1$, by Lemma 2.3, there exist constants $\beta>1$ and $C_{1}>0$, such that

$$
\|w\|_{\beta, B} \leq C_{1}|B|^{(1-\beta) / \beta}\|w\|_{1, B}
$$

for any cube or any ball $B \subset \mathbf{R}^{n}$. Let $s=n / \beta$. Then $\beta=n / s$. Since $1 / s=1 / n+(n-s) / n s$, by Hölder's inequality, Lemma 2.5 and (2.16), we have

$$
\begin{aligned}
& \left(\int_{B}\left|u-u_{B}\right|^{s} w d x\right)^{1 / s} \\
& \leq\left(\int_{B}\left(w^{1 / s}\right)^{n} d x\right)^{1 / n}\left(\int_{B}\left|u-u_{B}\right|^{n s /(n-s)} d x\right)^{(n-s) / n s} \\
& =C_{2}\|d u\|_{s, B}\left(\int_{B} w^{n / s} d x\right)^{1 / n} \\
& =C_{2}\|d u\|_{s, B} \cdot\|w\|_{\beta, B}^{1 / s} \\
& \leq C_{3}|B|^{(1-\beta) / s \beta}\|w\|_{1, B}^{1 / s} \cdot\|d u\|_{s, B} .
\end{aligned}
$$

Using Hölder's inequality again, we have

$$
\begin{aligned}
\|d u\|_{s, B} & =\left(\int_{B}\left(|d u| w^{1 / n} w^{-1 / n}\right)^{s} d x\right)^{1 / s} \\
& \leq\left(\int_{B}\left(|d u| w^{1 / n}\right)^{n} d x\right)^{1 / n}\left(\int_{B}\left(\frac{1}{w}\right)^{s /(n-s)} d x\right)^{(n-s) / n s} \\
& =\left(\int_{B}|d u|^{n} w d x\right)^{1 / n} \cdot\left\|(1 / w)^{1 / n}\right\|_{n s /(n-s), B}
\end{aligned}
$$


Combining (2.17) and (2.18) yields

$$
\begin{aligned}
& \left(\int_{B}\left|u-u_{B}\right|^{s} w d x\right)^{1 / s} \\
& \leq C_{3}|B|^{(1-\beta) / s \beta}\|w\|_{1, B}^{1 / s} \cdot\left\|(1 / w)^{1 / n}\right\|_{n s /(n-s), B} \cdot\left(\int_{B}|d u|^{n} w d x\right)^{1 / n} .
\end{aligned}
$$

Since $w \in A_{n / s}^{s / n}$, then

$$
\begin{aligned}
& \|w\|_{1, B}^{1 / s} \cdot\left\|(1 / w)^{1 / n}\right\|_{n s /(n-s), B} \\
& =\left(\int_{B} w d x\right)^{1 / s}\left(\int_{B}\left(\frac{1}{w}\right)^{s /(n-s)} d x\right)^{(n-s) / n s} \\
& =\left(\left(\int_{B} w d x\right)\left(\int_{B}\left(\frac{1}{w}\right)^{1 /(n / s-1)} d x\right)^{(s / n)(n / s-1)}\right)^{1 / s} \\
& \left.=\left(|B|^{1+(s / n)(n / s-1)}\left(\frac{1}{|B|} \int_{B} w d x\right)\left(\frac{1}{|B|} \int_{B}\left(\frac{1}{w}\right)^{1 /(n / s-1)} d x\right)^{(s / n)(n / s-1)}\right)^{1 / s}\right)^{1 / 2} \\
& \leq C_{4}|B|^{2 / s-1 / n} .
\end{aligned}
$$

Substituting (2.20) in (2.19) and using $n=s \beta$, we obtain

$$
\left(\int_{B}\left|u-u_{B}\right|^{s} w d x\right)^{1 / s} \leq C_{5}|B|^{1 / s}\left(\int_{B}|d u|^{n} w d x\right)^{1 / n}
$$

that is

$$
\left(\frac{1}{|B|} \int_{B}\left|u-u_{B}\right|^{s} w d x\right)^{1 / s} \leq C_{5}|B|^{1 / n}\left(\frac{1}{|B|} \int_{B}|d u|^{n} w d x\right)^{1 / n} .
$$

We have completed the proof of Theorem 2.14.

Theorem 2.21. Let $u \in D^{\prime}\left(B, \wedge^{l}\right)$ and $d u \in L^{n}\left(B, \wedge^{l+1}\right), l=0,1, \ldots, n$. If $1<s<n$ and $w \in A_{n / s}^{1}$, then there exists a constant $C$, independent of $u$ and $d u$, such that

$$
\left(\frac{1}{|B|} \int_{B}\left|u-u_{B}\right|^{s} w^{s / n} d x\right)^{1 / s} \leq C\left(\int_{B}|d u|^{n} w d x\right)^{1 / n}
$$

for any ball or any cube $B \subset \mathbf{R}^{n}$.

The proof of Theorem 2.21 is similar to that of Theorem 2.14. For completion of the paper, we prove Theorem 2.21 as follows. 
Proof. Since $1 / s=1 / n+(n-s) / n s$, by Hölder's inequality and Lemma 2.5, we have

$$
\begin{aligned}
& \left(\int_{B}\left|u-u_{B}\right|^{s} w^{s / n} d x\right)^{1 / s} \\
& \leq\left(\int_{B}\left(w^{1 / n}\right)^{n} d x\right)^{1 / n}\left(\int_{B}\left|u-u_{B}\right|^{n s /(n-s)} d x\right)^{(n-s) / n s} \\
& =\left\|w^{1 / n}\right\|_{n, B} \cdot C_{1}\|d u\|_{s, B} .
\end{aligned}
$$

Using Hölder's inequality again, we have

$$
\begin{aligned}
\|d u\|_{s, B} & =\left(\int_{B}\left(|d u| w^{1 / n} w^{-1 / n}\right)^{s} d x\right)^{1 / s} \\
& \leq\left(\int_{B}\left(|d u| w^{1 / n}\right)^{n} d x\right)^{1 / n}\left(\int_{B}\left(\frac{1}{w}\right)^{s /(n-s)} d x\right)^{(n-s) / n s} \\
& =\left(\int_{B}|d u|^{n} w d x\right)^{1 / n} \cdot\left\|(1 / w)^{1 / n}\right\|_{n s /(n-s), B}
\end{aligned}
$$

Combining (2.23) and (2.24) yields

$$
\begin{aligned}
& \left(\int_{B}\left|u-u_{B}\right|^{s} w^{s / n} d x\right)^{1 / s} \\
& \leq C_{1}\left\|w^{1 / n}\right\|_{n, B} \cdot\left\|(1 / w)^{1 / n}\right\|_{n s /(n-s), B} \cdot\left(\int_{B}|d u|^{n} w d x\right)^{1 / n} .
\end{aligned}
$$

Since $w \in A_{n / s}^{1}$, then

$$
\begin{aligned}
& \left\|w^{1 / n}\right\|_{n, B} \cdot\left\|(1 / w)^{1 / n}\right\|_{n s /(n-s), B} \\
& =\left(\left(\int_{B} w d x\right)\left(\int_{B}\left(\frac{1}{w}\right)^{s /(n-s)} d x\right)^{(n-s) / s}\right)^{1 / n} \\
& =\left(|B|^{n / s}\left(\frac{1}{|B|} \int_{B} w d x\right)\left(\frac{1}{|B|} \int_{B}\left(\frac{1}{w}\right)^{1 /(n / s-1)} d x\right)^{n / s-1}\right)^{1 / n} \\
& \leq C_{2}|B|^{1 / s}
\end{aligned}
$$

Substituting (2.26) in (2.25), we obtain

$$
\left(\int_{B}\left|u-u_{B}\right|^{s} w^{s / n} d x\right)^{1 / s} \leq C_{3}|B|^{1 / s}\left(\int_{B}|d u|^{n} w d x\right)^{1 / n},
$$


that is

$$
\left(\frac{1}{|B|} \int_{B}\left|u-u_{B}\right|^{s} w^{s / n} d x\right)^{1 / s} \leq C_{3}\left(\int_{B}|d u|^{n} w d x\right)^{1 / n} .
$$

We have completed the proof of Theorem 2.21.

\section{Global weighted integral inequalities.}

Susan G. Staples introduces the following $L^{s}$-averaging domains $[\mathbf{S}]$ : A proper subdomain $\Omega \subset \mathbf{R}^{n}$ is called an $L^{s}$-averaging domain, $s \geq 1$, if there exists a constant $\mathrm{C}$ such that

$$
\left(\frac{1}{|\Omega|} \int_{\Omega}\left|u-u_{\Omega}\right|^{s} d m\right)^{1 / s} \leq C \sup _{B \subset \Omega}\left(\frac{1}{|B|} \int_{B}\left|u-u_{B}\right|^{s} d m\right)^{1 / s}
$$

for all $u \in L_{l o c}^{s}(\Omega)$. Here $|\Omega|$ is the $n$-dimensional Lebesgue measure of $\Omega$. Susan G. Staples proves the Poincaré inequality in $L^{s}$-averaging domains in $[\mathbf{S}]$. In $[\mathbf{D N}]$, we introduce $L^{s}(\mu)$-averaging domains. We call a proper subdomain $\Omega \subset \mathbf{R}^{n}$ an $L^{s}(\mu)$-averaging domain, $s \geq 1$, if $\mu(\Omega)<\infty$ and there exists a constant $\mathrm{C}$ such that

$$
\left(\frac{1}{\mu\left(B_{0}\right)} \int_{\Omega}\left|u-u_{B_{0}}\right|^{s} d \mu\right)^{1 / s} \leq C \sup _{2 B \subset \Omega}\left(\frac{1}{\mu(B)} \int_{B}\left|u-u_{B}\right|^{s} d \mu\right)^{1 / s}
$$

for some ball $B_{0} \subset \Omega$ and all $u \in L_{\text {loc }}^{s}\left(\Omega ; \wedge^{l}\right)$. Here the measure $\mu$ is defined by $d \mu=w(x) d x$, where $w(x)$ is a weight and $w(x)>0$ a.e., and the supremum is over all balls $2 B \subset \Omega$.

Now we prove the following global weighted Poincaré-type inequality in $L^{s}(\mu)$-averaging domains.

Theorem 3.1. Let $u \in D^{\prime}\left(\Omega, \wedge^{l}\right)$ and $d u \in L^{p}\left(\Omega, \wedge^{l+1}\right), l=0,1, \ldots, n$. Assume that $s>1$ and $p>\max \{s, n\}$. Then exists a constant $\beta>1$ such that if $w \in A_{r}^{1} \cap A_{p / k}^{s / p}$, where $r>1, s \beta /(\beta-1) \leq k<p$ and $w \geq \eta>0$, we have

$$
\left(\frac{1}{\mu(\Omega)} \int_{\Omega}\left|u-u_{B_{0}}\right|^{s} w d x\right)^{1 / s} \leq C \mu(\Omega)^{1 / n}\left(\frac{1}{\mu(\Omega)} \int_{\Omega}|d u|^{p} w d x\right)^{1 / p}
$$

for any $L^{s}(\mu)$-averaging domain $\Omega$ and some ball $B_{0}$ with $2 B_{0} \subset \Omega$. Here the measure $\mu$ is defined by $d \mu=w(x) d x$ and $C$ is a constant independent of $u$ and $d u$.

Proof. Note

$$
\mu(B)=\int_{B} w d x \geq \int_{B} \eta d x=\eta|B|
$$

then

$$
|B| \leq C_{1} \mu(B)
$$


where $C_{1}=1 / \eta$. Since $p>n$, then $1 / n-1 / p>0$ and from (3.3) we have

$$
\begin{aligned}
\mu(B)^{-1 / s}|B|^{1 / s+1 / n-1 / p} & \leq \mu(B)^{-1 / s} \cdot\left(C_{1} \mu(B)\right)^{1 / s+1 / n-1 / p} \\
& =C_{2} \mu(B)^{1 / n-1 / p} \\
& \leq C_{2} \mu(\Omega)^{1 / n-1 / p} .
\end{aligned}
$$

By Theorem 2.6, the definition of $L^{s}(\mu)$-averaging domains and (3.4), we have

$$
\begin{aligned}
& \left(\frac{1}{\mu(\Omega)} \int_{\Omega}\left|u-u_{B_{0}}\right|^{s} d \mu\right)^{1 / s} \\
& \leq\left(\frac{1}{\mu\left(B_{0}\right)} \int_{\Omega}\left|u-u_{B_{0}}\right|^{s} d \mu\right)^{1 / s} \\
& \leq C_{3} \sup _{2 B \subset \Omega}\left(\frac{1}{\mu(B)} \int_{B}\left|u-u_{B}\right|^{s} d \mu\right)^{1 / s} \\
& =C_{3} \sup _{2 B \subset \Omega}\left(\left(\frac{|B|}{\mu(B)}\right)^{1 / s}\left(\frac{1}{|B|} \int_{B}\left|u-u_{B}\right|^{s} d \mu\right)^{1 / s}\right) \\
& \leq C_{3} \sup _{2 B \subset \Omega}\left(\left(\frac{|B|}{\mu(B)}\right)^{1 / s} \cdot C_{4}|B|^{1 / n}\left(\frac{1}{|B|} \int_{B}|d u|^{p} w d x\right)^{1 / p}\right) \\
& \leq C_{5} \sup _{2 B \subset \Omega}\left(\mu(B)^{-1 / s}|B|^{1 / s+1 / n-1 / p}\left(\int_{B}|d u|^{p} w d x\right)^{1 / p}\right) \\
& \leq C_{5} \sup _{2 B \subset \Omega}\left(C_{2} \mu(\Omega)^{1 / n-1 / p}\left(\int_{B}|d u|^{p} w d x\right)^{1 / p}\right) \\
& \leq C_{6} \sup _{2 B \subset \Omega}\left(\mu(\Omega)^{1 / n-1 / p}\left(\int_{\Omega}|d u|^{p} w d x\right)^{1 / p}\right)^{1 / p} \\
& =C_{6} \mu(\Omega)^{1 / n-1 / p}\left(\int_{\Omega}|d u|^{p} w d x\right)^{1 / p} \cdot \\
& =C_{6} \mu(\Omega)^{1 / n}\left(\frac{1}{\mu(\Omega)} \int_{\Omega}|d u|^{p} w d x\right)^{1 / p} \cdot
\end{aligned}
$$

Hence, we obtain

$$
\left(\frac{1}{\mu(\Omega)} \int_{\Omega}\left|u-u_{B_{0}}\right|^{s} w(x) d x\right)^{1 / s} \leq C \mu(\Omega)^{1 / n}\left(\frac{1}{\mu(\Omega)} \int_{\Omega}|d u|^{p} w d x\right)^{1 / p} .
$$

This completes the proof of Theorem 3.1.

Definition 3.6. We call $\Omega$, a proper subdomain of $\mathbf{R}^{n}, \delta$-John domain, $\delta>0$, if there exists a point $x_{0} \in \Omega$ which can be joined with any other 
point $x \in \Omega$ by a continuous curve $\gamma \subset \Omega$ so that

$$
d(\xi, \partial \Omega) \geq \delta|x-\xi|
$$

for each $\xi \in \gamma$. Here $d(\xi, \partial \Omega)$ is the Euclidean distance between $\xi$ and $\partial \Omega$.

As we know, John domains are bounded. Bounded quasiballs and bounded uniform domains are John domains. Also we know that a $\delta$-John domain has the following properties $[\mathbf{N}]$.

Lemma 3.7. Let $\Omega \subset \mathbf{R}^{n}$ be a $\delta$-John domain. Then there exists a covering $\mathcal{V}$ of $\Omega$ consisting of open cubes such that:

(i) $\sum_{Q \in \mathcal{V}} \chi_{\sigma Q}(x) \leq N \chi_{\Omega}(x), \quad \sigma>1$ and $x \in \mathbf{R}^{n}$.

(ii) There is a distinguished cube $Q_{0} \in \mathcal{V}$ (called the central cube) which can be connected with every cube $Q \in \mathcal{V}$ by a chain of cubes $Q_{0}, Q_{1}, \ldots, Q_{k}$ $=Q$ from $\mathcal{V}$ such that for each $i=0,1, \ldots, k-1$,

$$
Q \subset N Q_{i} .
$$

There is a cube $R_{i} \subset \mathbf{R}^{n}$ (this cube does not need to be a member of $\mathcal{V}$ ) such that

$$
R_{i} \subset Q_{i} \cap Q_{i+1} \text {, and } Q_{i} \cup Q_{i+1} \subset N R_{i} .
$$

We also know that if $w \in A_{r}^{1}$, then the measure $\mu$ defined by $d \mu=w(x) d x$ is a doubling measure, that is,

$$
\mu(2 B) \leq C \mu(B)
$$

for all balls $B$ in $\mathbf{R}^{n}$, see [HKM, p. 299]. Since the doubling property implies $\mu(B) \approx \mu(Q)$ whenever $Q$ is an open cube with $B \subset Q \subset \sqrt{n} B$, we may use cubes in place of balls whenever it is convenient to us.

Now we prove the following weighted global result in John domains.

Theorem 3.8. Let $u \in D^{\prime}\left(\Omega, \wedge^{l}\right)$ and $d u \in L^{n}\left(\Omega, \wedge^{l+1}\right), l=0,1, \ldots, n$. If $1<s<n$ and $w \in A_{n / s}^{1}$, then there exists a constant $C$, independent of $u$ and du, such that

$$
\left(\frac{1}{|\Omega|} \int_{\Omega}\left|u-u_{Q}\right|^{s} w^{s / n} d x\right)^{1 / s} \leq C\left(\int_{\Omega}|d u|^{n} w d x\right)^{1 / n}
$$

for any $\delta$-John domain $\Omega \subset \mathbf{R}^{n}$. Here $Q$ is any cube in the covering $\mathcal{V}$ of $\Omega$ appearing in Lemma 3.7.

Proof. We can write (2.22) as

$$
\int_{Q}\left|u-u_{Q}\right|^{s} w^{s / n} d x \leq C_{1}|Q|\left(\int_{Q}|d u|^{n} w d x\right)^{s / n},
$$


where $Q \subset \mathbf{R}^{n}$ is any cube. Suppose $\sigma>1$, by (3.9) and the condition i) in Lemma 3.7, we have

$$
\begin{aligned}
\int_{\Omega}\left|u-u_{Q}\right|^{s} w^{s / n} d x & \leq \sum_{Q \in \mathcal{V}} \int_{Q}\left|u-u_{Q}\right|^{s} w^{s / n} d x \\
& \leq C_{1} \sum_{Q \in \mathcal{V}}|Q|\left(\int_{Q}|d u|^{n} w d x\right)^{s / n} \\
& \leq C_{1}|\Omega| \sum_{Q \in \mathcal{V}}\left(\int_{\sigma Q}|d u|^{n} w d x\right)^{s / n} \\
& \leq C_{1}|\Omega| N\left(\int_{\Omega}|d u|^{n} w d x\right)^{s / n} \\
& =C_{2}|\Omega|\left(\int_{\Omega}|d u|^{n} w d x\right)^{s / n} .
\end{aligned}
$$

Thus, we have

$$
\left(\frac{1}{|\Omega|} \int_{\Omega}\left|u-u_{Q}\right|^{s} w^{s / n} d x\right)^{1 / s} \leq C_{3}\left(\int_{\Omega}|d u|^{n} w d x\right)^{1 / n}
$$

We have completed the proof of Theorem 3.8.

Applying Theorem 2.14 and using the same method that we used in the proof of Theorem 3.1, we have the following global result.

Theorem 3.10. Let $u \in D^{\prime}\left(\Omega, \wedge^{l}\right)$ and $d u \in L^{n}\left(\Omega, \wedge^{l+1}\right), l=0,1, \ldots, n$. Then there exists a constant $\beta>1$ such that if $w \in A_{r}^{1} \cap A_{n / s}^{s / n}$, where $s=n / \beta, r>1$ and $w \geq \eta>0$, we have

$$
\left(\frac{1}{\mu(\Omega)} \int_{\Omega}\left|u-u_{B_{0}}\right|^{s} w d x\right)^{1 / s} \leq C \mu(\Omega)^{1 / n}\left(\frac{1}{\mu(\Omega)} \int_{\Omega}|d u|^{n} w d x\right)^{1 / n}
$$

for any $L^{s}(\mu)$-averaging domain $\Omega$ and some ball $B_{0}$ with $2 B_{0} \subset \Omega$. Here the measure $\mu$ is defined by $d \mu=w(x) d x$ and $C$ is a constant independent of $u$ and $d u$.

Note that (3.11) is equivalent to

$$
\left(\frac{1}{\mu(\Omega)} \int_{\Omega}\left|u-u_{B_{0}}\right|^{s} d \mu\right)^{1 / s} \leq C\left(\int_{\Omega}|d u|^{n} d \mu\right)^{1 / n} .
$$

Proof. Since

$$
\mu(B)=\int_{B} w d x \geq \int_{B} \eta d x=\eta|B|
$$


then

$$
\frac{|B|}{\mu(B)} \leq C_{1}
$$

where $C_{1}=1 / \eta$. By Theorem 2.14, the definition of $L^{s}(\mu)$-averaging domains and (3.12), we have

$$
\begin{aligned}
& \left(\frac{1}{\mu(\Omega)} \int_{\Omega}\left|u-u_{B_{0}}\right|^{s} d \mu\right)^{1 / s} \\
& \leq\left(\frac{1}{\mu\left(B_{0}\right)} \int_{\Omega}\left|u-u_{B_{0}}\right|^{s} d \mu\right)^{1 / s} \\
& \leq C_{2} \sup _{2 B \subset \Omega}\left(\frac{1}{\mu(B)} \int_{B}\left|u-u_{B}\right|^{s} d \mu\right)^{1 / s} \\
& =C_{2} \sup _{2 B \subset \Omega}\left(\left(\frac{|B|}{\mu(B)}\right)^{1 / s}\left(\frac{1}{|B|} \int_{B}\left|u-u_{B}\right|^{s} d \mu\right)^{1 / s}\right) \\
& \leq C_{2} \sup _{2 B \subset \Omega}\left(\left(\frac{|B|}{\mu(B)}\right)^{1 / s} C_{3}\left(\int_{B}|d u|^{n} d \mu\right)^{1 / n}\right) \\
& \leq C_{4} \sup _{2 B \subset \Omega}\left(\int_{\Omega}^{\left.|d u|^{n} d \mu\right)^{1 / n}}\right. \\
& =C_{4}\left(\int_{\Omega}|d u|^{n} d \mu\right)^{1 / n} .
\end{aligned}
$$

Thus, we have

$$
\left(\frac{1}{\mu(\Omega)} \int_{\Omega}\left|u-u_{B_{0}}\right|^{s} d \mu\right)^{1 / s} \leq C\left(\int_{\Omega}|d u|^{n} d \mu\right)^{1 / n} .
$$

We have completed the proof of Theorem 3.10.

Remark. Since $L^{s}(\mu)$-averaging domains reduce to $L^{s}$-averaging domains if $w=1$ ( $\operatorname{so} d \mu=w(x) d x=d x$ ), then Theorem 3.1 and Theorem 3.10 also hold if $\Omega \subset \mathbf{R}^{n}$ is an $L^{s}$-averaging domain.

\section{References}

[B] J.M. Ball, Convexity conditions and existence theorems in nonlinear elasticity, Arch. Rational Mech. Anal., 63 (1977), 337-403.

[C] H. Cartan, Differential forms, Houghton Mifflin, Co., Boston, 1970.

[D1] Shusen Ding, Weighted Hardy-Littlewood inequality for A-harmonic tensors, Proc. Amer. Math. Soc., 125 (1997), 1727-1735.

[D2] Integrability of conjugate A-harmonic tensors in $L^{s}(\mu)$-averaging domains, preprint. 
[DN] Shusen Ding and Craig Nolder, $L^{s}(\mu)$-averaging domains and their applications, preprint.

[G] J.B. Garnett, Bounded Analytic Functions, New York, Academic Press, 1970.

[HKM] Juha Heinonen, Tero Kilpelainen and Olli Martio, Nonlinear potential theory of degenerate elliptic equations, Oxford, 1993.

[I] T. Iwaniec, p-harmonic tensors and quasiregular mappings, Annals of Mathematics, 136 (1992), 589-624.

[IL] T. Iwaniec and A. Lutoborski, Integral estimates for null Lagrangians, Arch. Rational Mech. Anal., 125 (1993), 25-79.

[IM] T. Iwaniec and G. Martin, Quasiregular mappings in even dimensions, Acta Math., 170 (1993), 29-81.

[N] C.A. Nolder, Hardy-Littlewood theorems for A-harmonic tensors, Illinois Journal of Mathematics, to appear.

[S] Susan G. Staples, $L^{p}$-averaging domains and the Poincare inequality, Ann. Acad. Sci. Fenn, Ser. AI Math., 14 (1989), 103-127.

Received July 21, 1997.

SEATtLE UNIVERSity

SEATtLE, WA 98122

E-mail address: sding@seattleu.edu 